\title{
Associations between maternal BMI, breastfeeding practices and infant anthropometric status in Colombia; secondary analysis of ENSIN 2010
}

\author{
Fanny Aldana-Parra ${ }^{*^{*}}$ D, Gilma Olaya Vega ${ }^{1}$ and Mary Fewtrell ${ }^{2}$
}

\begin{abstract}
Background: Maternal malnutrition and infant feeding mode impact short and long term infant and child morbidity and mortality. The period of lactation may provide an opportunity to modulate the risk of disease later in life. Our aim was to estimate the effect of maternal body mass index (BMI) and infant feeding mode, particularly breastfeeding practices, on the anthropometric status of children under 2 years in Colombia.

Methods: A secondary analysis was performed using the data from ENSIN 2010. Term infants under 2y, singleton, with a mother older than 18y, were included in the analysis. Outcomes were wasting (WLZ <-2SD), overweight $(W L Z>+2 S D)$ and stunting (LAZ <-2SD). Predictors were infant feeding (exclusive and predominant BF constructed from 24-h recall, age at introduction of liquids, semisolids and solids) and maternal BMI. Socioeconomic variables, maternal education and age, conditions during pregnancy and birth weight were analyzed as covariates.

Results: Mothers of overweight infants had higher BMI (Mean dif $=1.47 \mathrm{~kg} / \mathrm{m}^{2} ; 95 \% \mathrm{Cl}=2.1,0.8$ ) than those with normal weight infants. Stunting and wasting were not predicted by maternal anthropometry or infant feeding mode. Fewer maternal years of education were associated with wasting $(\mathrm{OR}=0.90 ; 95 \% \mathrm{Cl}=0.86,0.97 ; p=0.003)$ and stunting $(\mathrm{OR}=0.92 ; 95 \% \mathrm{Cl}=0.89,0.94 ; p<0.0001)$, while more maternal years of education were associated with overweight $(\mathrm{OR}=1.06 ; 95 \% \mathrm{Cl}=1.02,1.01 ; p=0.001)$; higher birth weight was associated with overweight $(\mathrm{OR}=1.001 ; 95 \% \mathrm{Cl}=1.00,1.001 ; p<0.0001)$ and lower birth was associated with stunting $(\mathrm{OR}=0.99 ; 95 \% \mathrm{Cl}=0.89$; $p<0.0001)$ in the final regression model.
\end{abstract}

Conclusions: Maternal BMI is a modifiable target for public health policy to promote healthy infant growth. Infant nutritional status is affected by direct and indirect factors that need to be addressed in further studies.

Keywords: Maternal nutritional status, Lactating women, Infant nutritional status, Maternal-infant health, Colombian population, Stunting, Wasting, Maternal overweight, Maternal undernutrition, Children overweight, Breastfeeding, Exclusive breastfeeding

\footnotetext{
* Correspondence: aldanafanny@javeriana.edu.co

'Departamento de Nutrición y Bioquímica, Pontificia Universidad Javeriana,

Bogotá, Colombia

Full list of author information is available at the end of the article
}

(c) The Author(s). 2020 Open Access This article is distributed under the terms of the Creative Commons Attribution 4.0 International License (http://creativecommons.org/licenses/by/4.0/), which permits unrestricted use, distribution, and reproduction in any medium, provided you give appropriate credit to the original author(s) and the source, provide a link to the Creative Commons license, and indicate if changes were made. The Creative Commons Public Domain Dedication waiver (http://creativecommons.org/publicdomain/zero/1.0/) applies to the data made available in this article, unless otherwise stated. 


\section{Background}

Maternal malnutrition negatively impacts infant and child morbidity and mortality. The Developmental Origins of Health and Disease Hypothesis considers the periconceptional period as a window of opportunity to influence long term body composition and endocrine characteristics of the offspring [1,2]. Early human observational studies suggested that maternal famine, especially during periods of war, increases the risk of cardiometabolic diseases in the offspring as adults [3]. Maternal obesity could also have a detrimental effect on the development of obesity in offspring, as shown in observational studies [4].

The period of lactation may provide an opportunity to modulate the impact of genetic and prenatal factors experienced by the fetus [5]. Animal models show that pups fed with a cafeteria diet during gestation and lactation had increased adiposity at weaning, which could predispose to metabolic disorders later in life [6]. In humans, formula feeding during the first months of life can result in accelerated weight gain. In a large population study $(n=5560)$, children formula-fed since birth were more likely to be obese at school age $(\mathrm{OR}=1.57$; 95\% CI 1.2-2.2) [7]. Breastfeeding (BF) is associated with protection against respiratory and gastrointestinal infections [8], a positive effect on cognitive and neurological development [9] and reduction of breast, endometrial and ovarian cancer for the mother [10-12]. Other possible but not well-established benefits for the infant include a lower risk of obesity, diabetes [13] and hypertension [14]. However, EBF prevalence during the first 6 months in middle-income countries is around $37 \%$ [15], and in Colombia, this decreased from $46.9 \%$ in 2005 to $36.1 \%$ in 2015 [16].

While global mortality in children under 5 years fell from 93 per 1000 live births in 1990 to 41 per 1000 live births in 2016 [17], and the prevalence of stunting decreased from $39.2 \%$ in 1990 to $21.9 \%$ in 2018 [18], the prevalence of obesity increased in childbearing women and children under 5 years. In 2015, the worldwide prevalence of obesity in women over 18 years was $15.2 \%$, and in children under 5 years, 5.9\% were overweight, $22 \%$ stunted and $7.5 \%$ wasted during 2017 [19]. During 2015, Colombia, a Latin-American country ranked by World Bank in the upper-middle-income group [20], reported a prevalence of obesity in childbearing women and children under 5 years of 22.4 and $6.3 \%$, respectively and a prevalence of stunting of $10.8 \%$ and wasting of 2.3\% during the same year (Encuesta Nacional de la Situación Nutricional (ENSIN)) [16].

Considering maternal nutritional status and breastfeeding practices as potential factors that could influence children nutritional status [21, 22], and given the lack of information in Colombia about the associations between these factors and infant stunting, wasting and overweight, the purpose of this secondary analysis using data from ENSIN 2010 was to estimate the effect of maternal body mass index (BMI) and infant feeding mode, particularly breastfeeding (BF) practices, on the anthropometric status of children under 2 years in Colombia.

\section{Methods}

\section{Study design}

This study was a secondary analysis of a national survey conducted in Colombia during 2010: Encuesta Nacional de la Situación Nutricional en Colombia (ENSIN) [23]. The ENSIN is a survey of households with national coverage and urban and rural representation, which includes sociodemographic characteristics of the households, mother and infant anthropometric data, and information about breastfeeding and early feeding. The dataset was provided by the program Demographic Health Survey (DHS) belonging to the United States Agency for International Development (USAID), which aims to collect, analyze and disseminate accurate and representative data on the characteristics of the population, health and nutrition in countries around the world.

\section{Population and sample}

The population of Colombia during 2010 was about 45, 510,000 based on estimates from the national population census ( $n=23,277,201$ female; 50.69\%) [24]. The ENSIN 2010 is a descriptive and cross-sectional survey that describes the Colombian sociodemographic and nutritional situation, in a population from birth to 64 years old in 258 municipalities, both rural and urban. The ENSIN sample was randomly selected from the national census of 2005, whose calculation was based on the estimation of global malnutrition according to previous national surveys (ENSIN and ENDS 2005) [25]; the methodological design of ENSIN 2010 is described elsewhere [23]. It included 17,756 children under 5 years. For the present analysis, inclusion and exclusion criteria were:

a) Inclusion criteria: Infant under 2 years $(n=7002)$ with gestational age at birth $\geq 37$ weeks.

b) Exclusion criteria: Infant from multiple pregnancy $(n=100 ; 1.43 \%)$, pregnancy during the survey $(n=$ $250 ; 3.57 \%)$, gestational age at birth less than 36 weeks ( $n=703 ; 10.03 \%)$ and infant with implausible weight and/or length ( \pm 5 SD of weight for length) $(n=248 ; 4.33 \%)$.

Mothers above 18 years who were not pregnant at the time the survey and who were the respondent of the survey were included. If more than one child under $5 y$ was living in a single house, the youngest was included in the survey. 


\section{Data collection}

Baseline characteristics, maternal and infant anthropometry and $\mathrm{BF}$ and other infant feeding mode data were collected using validated questionnaires in the house of the interviewee. Trained dietitians administered the questionnaires and made infant and maternal anthropometric measurements; all data collection procedures and performance of the interviewers were tested in a pilot study and standardized through procedure manuals. Dietitians participated in a two-month training course for data collection including administration of the questionnaires. The dietitian recorded data in the participant's house using a portable device with CSPro software, which can detect inconsistencies. The mother provided information about breastfeeding for the youngest child aged under 5y living in the house.

a) Measurement of anthropometry Infant and maternal weight were measured with a SECA 872 digital scale (capacity of $200 \mathrm{~kg}$ and accuracy of $50 \mathrm{~g}$ ), with minimum clothing or other items that could alter the weight as recommended by WHO [26]. Infant and mother were weighed together, and infant weight was calculated by subtracting the weight of both minus the maternal weight. Maternal height (standing) and infant supine length were taken with portable wooden column scale, suitable for infants and adults, without shoes or ornaments on the head that could interfere with the measurement [26].

b) Measurement of predictors of infant anthropometry Information about breastfeeding practices during the last $24 \mathrm{~h}$ was recorded using a questionnaire. For the present analysis, we selected only information about infants under 2 years.

\section{Study variables}

a) Outcome: Infant anthropometry

Weight for length Z-score (WLZ) and length for age Z-score (LAZ) reflect infant anthropometric status and were calculated using the WHO growth standards [27]. For the present study, infant stunting was defined as LAZ $<-2$ Standard Deviations (SD), wasting as $\mathrm{WLZ}<-2 \mathrm{SD}$ and overweight as WLZ $>+2$ SD as recommended by WHO [28].

b) Predictors of infant anthropometry Maternal anthropometric status. Maternal BMI was calculated as the ratio between maternal weight in $\mathrm{kg}$ divided by maternal height squared, and analysed as a continuous variable.

Breastfeeding practices and infant feeding mode variables were evaluated based on WHO indicators: age at initiation of BF, age at introduction of other liquids, semi-solid and solid foods, duration of BF, EBF and predominant BF [29]. EBF was defined as the infant currently being $\mathrm{BF}$ and not having consumed other liquids, semisolid or solid food in the last $24 \mathrm{~h}$; EBF information was only available for infants aged under 6 months at the time of the survey. Predominant BF was defined as infants currently BF with consumption of other liquids less than three times in the last $24 \mathrm{~h}$, excluding infants with EBF. Duration of BF was recorded for infants who had already stopped BF. Inconsistencies in infant feeding data, for example, a duration of BF longer than the age of the infant at the time of the survey, were registered as missing values. We considered infant formula feeding separately as a baseline characteristic and as a possible covariate in analyses predicting infant anthropometry.

c) Other variables:

Sociodemographic variables such as place of residence (urban/rural) and wealth index (poorer/ poorest, middle and richer/richest) [30] were recorded. Other maternal variables were maternal age, ethnicity (mestizo/minorities), education attainment in years, living with the partner, currently working, alcohol and cigarettes consumption during pregnancy and delivery by caesarean section. Infant variables also included birth weight, gender and age in months. Paternal anthropometric status and parental adiposity, which have been reported to be associated with infant nutritional status in other studies [31,32], were not analysed due to a lack of information in the survey. ENSIN 2010 did not specify if the mother's current partner was the father of the infant.

\section{Statistical analysis}

Descriptive analysis of baseline characteristics is presented as prevalences for categorical variables and mean and standard deviation for continuous variables; WLZ was categorized as wasting, normal and overweight and LAZ as stunting or normal. Comparisons between groups were made with t-test, ANOVA, Kruskal Wallis, U-Mann Witney or $\chi^{2}$ test as appropriate and the OR and mean difference with the corresponding 95\% CI were given. For variables with more than three categories pairwise post hoc comparisons were made using the Bonferroni test. Binary and multinomial logistic regression models were used to examine predictors of wasting, overweight and stunting. The univariate analysis identified significant associations associations of maternal BMI and/or infant feeding mode variables with wasting, overweight and stunting; and then, these were included in the multivariable models (unadjusted, and then subsequently also adjusted for the other covariates). All 


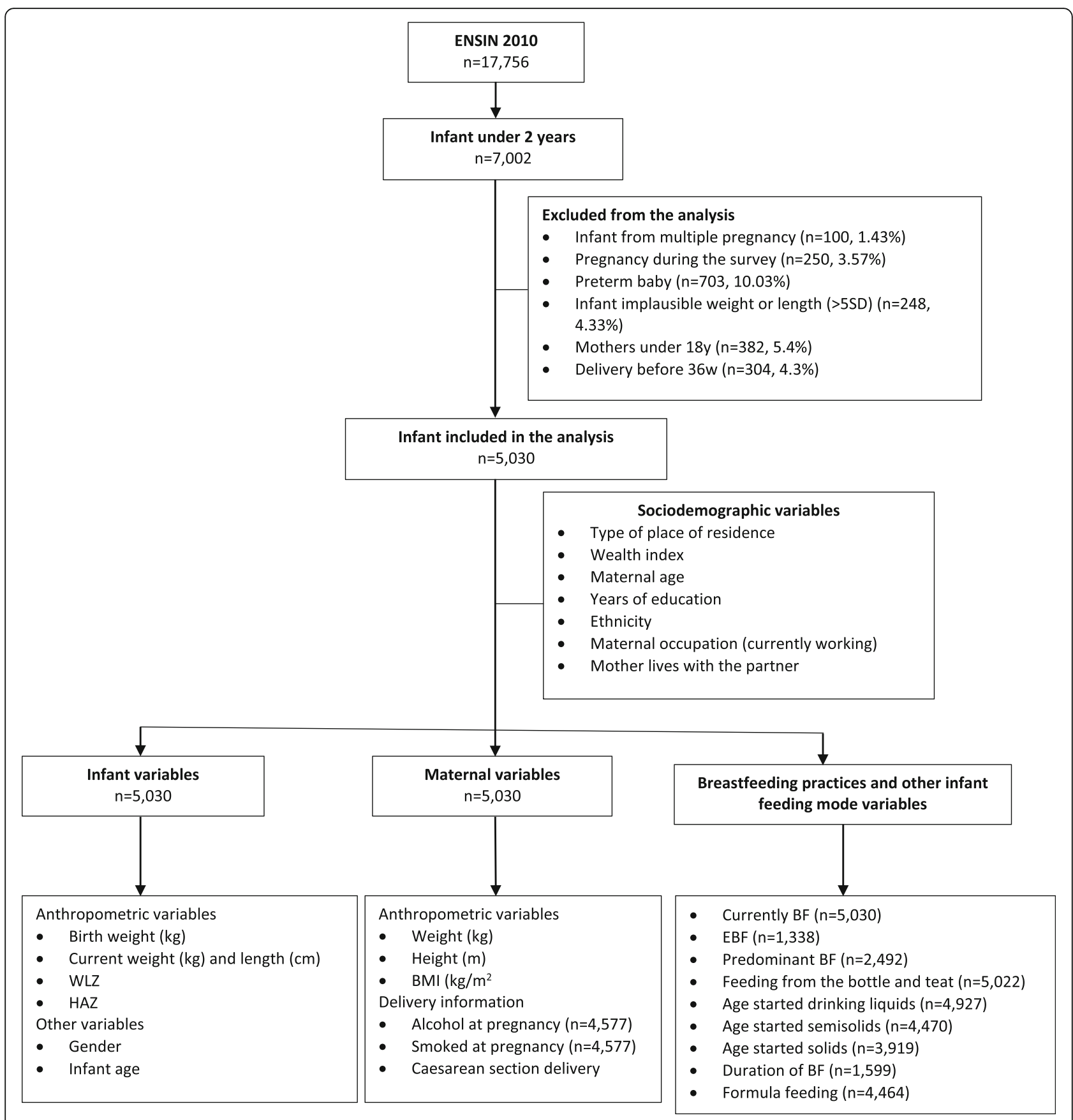

Fig. 1 Sample analysed and study variables flow diagram. Secondary analysis, ENSIN 2010

comparisons and regressions had normal WLZ and LAZ as the reference group. Statistical analysis was performed with IBM SPSS version 24.

\section{Ethical aspects}

Resolution 8430 addresses ethical research aspects in Colombia. All families participating in the ENSIN 2010 gave informed consent after receiving information about survey [23]. For this analysis, the permits were requested from the Instituto Colombiano de Bienestar Familiar (ICBF) and the DHS program for the use of the databases necessary for the analysis.

\section{Results}

An overall sample of $n=5030$ mother-infant dyads with complete anthropometric measures was analysed. Information collected in the survey about EBF (only in infants under 6 months), current BF and predominant BF were 
Table 1 Baseline characteristics compared between WLZ categories, ENSIN 2010

\begin{tabular}{|c|c|c|c|c|c|c|c|}
\hline & \multirow{2}{*}{$\begin{array}{l}\text { Overall } \\
(n=5030)\end{array}$} & \multicolumn{6}{|l|}{ WLZ } \\
\hline & & $\begin{array}{l}\text { Wasting } \\
<-2 \text { SD } \\
(n=74)\end{array}$ & $\begin{array}{l}\text { Normal } \\
\geq-2 \mathrm{SD} \\
\leq+2 \mathrm{SD} \\
(n=4663)\end{array}$ & $\begin{array}{l}\text { Overweight } \\
>+2 \mathrm{SD} \\
(n=293)\end{array}$ & $\begin{array}{l}\text { OR } \\
\text { /Difference }\end{array}$ & $95 \% \mathrm{Cl}$ & $p$ value \\
\hline \multicolumn{8}{|l|}{ Sociodemographic characteristics (n, \%) } \\
\hline \multicolumn{8}{|l|}{ Type of place of residence } \\
\hline Rural & $1841(36.6)$ & $27(36.5)$ & $1706(36.6)$ & $108(36.9)$ & $0.98^{b}$ & $0.77,1.26$ & 0.92 \\
\hline Urban & $3189(63.4)$ & $47(63.5)$ & $2957(63.4)$ & $185(63.1)$ & $0.99^{a}$ & $0.62,1.60$ & 0.98 \\
\hline \multicolumn{8}{|l|}{ Wealth index } \\
\hline \multirow[t]{2}{*}{ Poorer and poorest } & $3167(63)$ & $54(73)$ & $2937(63)$ & $176(60.1)$ & $1.35^{\mathrm{a}}$ & $0.72,2.53$ & 0.35 \\
\hline & & & & & $1.03^{b}$ & $0.75,1.43$ & 0.82 \\
\hline Middle & $945(18.8)$ & $12(16.2)$ & $882(18.9)$ & $51(17.4)$ & 1 & & \\
\hline \multirow[t]{2}{*}{ Richer and richest } & $918(18.3)$ & $8(10.8)$ & $844(18.1)$ & $66(22.5)$ & $0.54^{\mathrm{a}}$ & $0.26,1.13$ & 0.13 \\
\hline & & & & & $1.35^{b}$ & $0.92,1.97$ & 0.12 \\
\hline \multicolumn{8}{|l|}{ Maternal characteristics } \\
\hline \multirow[t]{2}{*}{ Age (y) (mean, SD) } & $26.6,( \pm 6.3)$ & $28( \pm 6.3)$ & $26.6( \pm 6.3)$ & $26.3( \pm 6.1)$ & $-1.4^{\mathrm{a}}$ & $-3.2,0.35$ & 0.16 \\
\hline & & & & & $0.3^{b}$ & $-0.61,1.21$ & 0.99 \\
\hline \multirow[t]{2}{*}{ Maternal education (y) (mean, SD) } & $8.4,( \pm 3.9)$ & $6.9( \pm 4.3)$ & $8.3( \pm 3.9)$ & $9.1( \pm 3.6)$ & $1.4^{\mathrm{a}}$ & $0.31,2.54$ & $0.01^{*}$ \\
\hline & & & & & $-0.8^{b}$ & $-1.4,-0.25$ & $0.002^{*}$ \\
\hline \multicolumn{8}{|l|}{ Ethnicity (n, \%) } \\
\hline Minorities & $1406(28)$ & $22(29.7)$ & $1294(27.8)$ & $90(30.7)$ & $0.92^{a}$ & $0.55,1.51$ & 0.69 \\
\hline Mestizo & $3624(72)$ & $52(70.3)$ & $3369(72.2)$ & $203(69.3)$ & $1.15^{\mathrm{b}}$ & $0.89,1.49$ & 0.27 \\
\hline \multicolumn{8}{|l|}{ Currently working (n, \%) } \\
\hline Yes & $2047(40.7)$ & $29(39.2)$ & $1888(40.5)$ & $130(44.4)$ & $0.94^{a}$ & $0.59,1.50$ & 0.81 \\
\hline No & $2983(59.3)$ & $45(60.8)$ & $2775(59.5)$ & $163(55.6)$ & $1.17^{\mathrm{b}}$ & $0.92,1.48$ & \\
\hline \multicolumn{8}{|l|}{ Mother lives with the partner $(n, \%)$} \\
\hline Yes & $3816(75.9)$ & $56(75.7)$ & $3527(75.6)$ & $233(79.5)$ & $0.99^{a}$ & $0.58,1.69$ & 0.53 \\
\hline No & $1214(24.1)$ & $18(24.3)$ & $1136(24.4)$ & $60(20.5)$ & $1.25^{b}$ & $0.93,1.67$ & 0.13 \\
\hline \multicolumn{8}{|l|}{ Pregnancy conditions } \\
\hline Alcohol at pregnancy $(n=4577)(n, \%)$ & & $n=74$ & $n=4577$ & $n=291$ & & & \\
\hline Yes & $451(9)$ & $6(8.1)$ & $412(9)$ & $33(11.3)$ & $0.87^{a}$ & $0.38,2.03$ & 0.48 \\
\hline No & $4491(89.3)$ & $68(91.9)$ & $4165(91)$ & $258(88.7)$ & $1.29^{b}$ & $0.88,1.88$ & 0.18 \\
\hline Smoked at pregnancy $(n=4577)(n, \%)$ & & $n=74$ & $n=4577$ & $n=291$ & & & \\
\hline Yes & $105(2.1)$ & $3(4.1)$ & $95(2.1)$ & $7(2.4)$ & $1.97^{\mathrm{a}}$ & $0.61,6.37$ & 0.21 \\
\hline No & $4837(96.2)$ & $71(95.9)$ & $4482(97.9)$ & $284(97.6)$ & $1.16^{b}$ & $0.53,2.52$ & 0.70 \\
\hline \multicolumn{8}{|l|}{ Caesarean section delivery $(\mathrm{n}, \%)$} \\
\hline Yes & $1579(31.4)$ & $21(28.4)$ & $1441(30.9)$ & 117 (39.9) & $0.86^{\mathrm{a}}$ & $0.52,1.44$ & 0.62 \\
\hline No & $3451(68.6)$ & $53(71.6)$ & $3222(69.1)$ & $176(60.1)$ & $1.49^{b}$ & $1.17,1.89$ & $<0.001^{* *}$ \\
\hline \multicolumn{8}{|l|}{ Infant caractetistics } \\
\hline \multirow[t]{3}{*}{ Birth weight $(\mathrm{kg})(n=3877)$ (mean, SD) } & & $n=53$ & $n=3585$ & $n=239$ & & & \\
\hline & $3.3,( \pm 0.5)$ & $3.2( \pm 0.6)$ & $3.3( \pm 0.5)$ & $3.4( \pm 0.5)$ & $0.08^{a}$ & $-0.08,0.25$ & 0.68 \\
\hline & & & & & $-0.17^{b}$ & $-0.25,-0.09$ & $0.001^{* *}$ \\
\hline \multicolumn{8}{|l|}{ Gender (n, \%) } \\
\hline Male & $2605(51.8)$ & $40(54.1)$ & $2416(51.8)$ & $149(50.9)$ & $0.91^{\mathrm{a}}$ & $0.57,1.44$ & 0.73 \\
\hline Female & $2425(48.2)$ & 34 (45.9) & $2247(48.2)$ & $144(49.1)$ & $0.96^{b}$ & $0.76,1.21$ & 0.75 \\
\hline
\end{tabular}


Table 1 Baseline characteristics compared between WLZ categories, ENSIN 2010 (Continued)

\begin{tabular}{|c|c|c|c|c|c|c|c|}
\hline & \multirow{3}{*}{$\begin{array}{l}\text { Overall } \\
(n=5030)\end{array}$} & \multicolumn{6}{|l|}{ WLZ } \\
\hline & & \multirow{2}{*}{$\begin{array}{l}\text { Wasting } \\
<-2 \text { SD } \\
(n=74)\end{array}$} & \multirow{2}{*}{$\begin{array}{l}\text { Normal } \\
\geq-2 S D \\
\leq+2 S D \\
(n=4663)\end{array}$} & \multirow{2}{*}{$\begin{array}{l}\text { Overweight } \\
>+2 \mathrm{SD} \\
(n=293)\end{array}$} & OR & $95 \% \mathrm{Cl}$ & $p$ value \\
\hline & & & & & \multicolumn{3}{|l|}{ /Difference } \\
\hline \multirow[t]{2}{*}{ Infant age (months) (mean, SD) } & \multirow[t]{2}{*}{$12.1,( \pm 7.1)$} & \multirow[t]{2}{*}{$8.9( \pm 6.9)$} & \multirow[t]{2}{*}{$12.3( \pm 7.1)$} & \multirow[t]{2}{*}{$11.1( \pm 6.9)$} & $3.39^{a}$ & $1.41,5.37$ & $0.001^{* *}$ \\
\hline & & & & & $1.14^{b}$ & $0.12,2.15$ & $0.02^{*}$ \\
\hline
\end{tabular}

Comparisons performed with $x^{2}$ test or Kruskall-Wallis test (all the continuous variables non-parametric distributed) as appropriate

${ }^{a}$ Comparisons between normal and wasting

${ }^{\mathrm{b}}$ Comparisons between normal and overweight

* Significance at the level of $p<0.05$

** Significance at the level of $p<0.001$

available for $n=1338, \mathrm{n}=5030$ and $n=2492$ subjects respectively (Fig. 1). 63.4\% of the study population lived in urban areas and $63 \%$ were in the lowest socioeconomic group. The mean maternal age was 26.6 years $( \pm 6.6)$ and the mean maternal years of education were $8.4( \pm 3.9)$. The majority of the sample were mestizo $(72 \%)$, not working (59.3\%) and living with the partner (75.9\%). During pregnancy, 9\% consumed alcohol and 2.1\% smoked. Caesarean section deliveries accounted for around one-third of the sample (31.4\%). The mean birth weight and infants ages were $3.3 \mathrm{~kg}( \pm 0.5)$ and 12.1 months $( \pm 7.1)$, respectively. $51.8 \%$ of the infants were male (Table 1).

\section{Infant anthropometric status, maternal BMI and breastfeeding practices}

The prevalence of wasting, overweight and stunting was $1.5 \%(n=74 / 5030), 5.8 \%(n=293 / 5030)$ and $12.8 \%(n=$ $645 / 5030)$ respectively. Mean maternal weight, height and BMI was $59.8 \pm 12 \mathrm{~kg}, 1.55 \pm 0.06 \mathrm{~m}$ and $24.8 \pm 4.6$ $\mathrm{kg} / \mathrm{m}^{2}$ respectively. EBF prevalence in infants under 6 months was $34.4 \%$; other infant feeding mode variables analysed were current BF (68\%), predominant BF (5.1\%), duration of $\mathrm{BF}$ (mean $=7.9 \pm 5.1$ months), age of initiation of liquids, semisolids and solids $(2.6 \pm 2.5$ months, $4.9 \pm 2.1$ months and $7.5 \pm 2.5$ months, respectively) and formula feeding (42\%) (Table 2).

\section{Predictors of wasting}

The bivariate analysis showed that wasted infants were more likely not to be currently $\mathrm{BF}(\mathrm{OR}=0.19 ; 95 \% \mathrm{CI}=$ $0.14,0.27)$, to have an earlier age of introduction of liquids (Mean dif. $=0.75$ months; $95 \% \mathrm{CI}=0.06,1.45$ ) and a later age of introduction of semisolids (Mean dif. $=0.71$ months; $95 \% \mathrm{CI}=0.05,1.37$ ) when compared with infants with normal WLZ. Wasting was not predicted by maternal anthropometry, EBF or predominant BF variables; although there was a trend for infants under 6 months who were not EBF to have a higher prevalence of wasting ( $n=19,66.1 \%$ in non-EBF vs $n=15,44.1 \%$ in $\mathrm{EBF})$. The mean duration of BF was not significantly different between those who were wasted and those who were not $(6.9$ months $\mathrm{v} 8.7$ months, mean dif. $=3.3$ months; $95 \% \mathrm{CI}=-0.2,6.81)$. Regarding baseline characteristics, infants with wasting had mothers with fewer years of education (Mean dif $=1.4 ; 95 \% \mathrm{CI}=0.31,2.54$ ), and mothers with lower age (Mean dif $=3.39 ; 95 \% \mathrm{CI}=$ 1.41, 5.37) (Table 2). Wealth index, a potential predictor of wasting, was not significantly associated; however, there was a trend for the poorer/poorest category to have a higher prevalence of wasting when compared with normal WLZ and overweight $(n=54,73 \%$ for wasting; $n=2937,63 \%$ for normal WLZ and $n=176,60.1 \%$ for overweight) (Table 1). The unadjusted logistic regression model showed that current BF, age of introduction of liquids and semisolids were not significant predictors of wasting. In the final model, the only significant predictor of wasting was fewer years of maternal education $(\beta=-0.09 ; \mathrm{OR}=0.91 ; 95 \% \mathrm{CI}=0.86,0.97)$; however, the model only predicted 0.4 to $1 \%$ of wasting in this population (Table 3).

\section{Predictors of overweight}

Higher maternal BMI and higher maternal weight were significantly different (Mean dif $=1.5 \mathrm{~kg} / \mathrm{m}^{2} ; 95 \% \mathrm{CI}=$ $2.1,0.8$ and $4.1 \mathrm{~kg} ; 95 \% \mathrm{CI}=5.8,2.3$, respectively) between infants with normal WLZ and those who were overweight, indicating a positive association between maternal BMI and weight, and infant overweight. Breastfeeding practices and other infant feeding mode variables were not significant predictors (Table 2). Overweight was also associated with more years of maternal education (Mean dif $=0.8$ years; $95 \% \mathrm{CI}=1.4,0.25$ ), vaginal delivery $(\mathrm{OR}=1.49 ; 95 \% \mathrm{CI}=1.2,1.9)$, higher birth weight (Mean dif $=0.17 \mathrm{~kg} ; 95 \% \mathrm{CI}=0.25,0.09)$ and lower infant age (Mean dif $=-1.14$ months; $95 \% \mathrm{CI}=-0.12$, 2.1) (Table 1).

Higher maternal BMI was significantly related to infant overweight in the unadjusted $(\beta=0.06$; $O R=1.06$; $95 \% \mathrm{CI}=1.03,1.08)$, adjusted $(\beta=0.05 ; \mathrm{OR}=1.05 ; 95 \%$ $\mathrm{CI}=1.02,1.08)$ and final model $(\beta=0.05 ; \mathrm{OR}=1.05 ; 95 \%$ $\mathrm{CI}=1.03,1.08)$. The adjusted model showed that more 
Table 2 Maternal anthropometry and breastfeeding practices compared between WLZ categories, ENSIN 2010

\begin{tabular}{|c|c|c|c|c|c|c|c|}
\hline & \multirow{2}{*}{$\begin{array}{l}\text { Overall } \\
(n=5030)\end{array}$} & \multicolumn{6}{|l|}{ WLZ } \\
\hline & & $\begin{array}{l}\text { Wasting <-2 } \\
\text { SD }(n=74)\end{array}$ & $\begin{array}{l}\text { Normal } \geq-2 \mathrm{SD} \\
\leq+2 \mathrm{SD}(n=4663)\end{array}$ & $\begin{array}{l}\text { Overweight }>+2 \\
\text { SD }(n=293)\end{array}$ & $\begin{array}{l}\text { OR } \\
\text { /Difference }\end{array}$ & $95 \% \mathrm{Cl}$ & $p$ value \\
\hline \multicolumn{8}{|l|}{ Maternal anthropometry (mean, median, SD) } \\
\hline \multirow[t]{3}{*}{ Weight $(\mathrm{kg})(n=4750)$} & & $n=165$ & $n=4496$ & $n=89$ & & & \\
\hline & $\begin{array}{l}59.8,57.6 \\
( \pm 12.0)\end{array}$ & $\begin{array}{l}53.9,52.5 \\
( \pm 10.9)\end{array}$ & $59.9,57.7( \pm 11.9)$ & $67.8,65.8( \pm 12.7)$ & $1.35^{\mathrm{a}}$ & $-2.2,4.9$ & 0.99 \\
\hline & & & & & $-4.1^{b}$ & $-5.8,-2.3$ & $<0.001^{* *}$ \\
\hline \multirow[t]{3}{*}{ Height $(\mathrm{mt})(n=4749)$} & & $n=165$ & $n=4495$ & $\mathrm{n}=89$ & & & \\
\hline & $\begin{array}{l}1.55,1.55 \\
( \pm 6.3)\end{array}$ & $\begin{array}{l}1.51,1.50 \\
( \pm 6.3)\end{array}$ & $1.55,1.55( \pm 6.2)$ & $1.57,1.57( \pm 5.5)$ & $0.72^{a}$ & $-2.58,1.13$ & 0.99 \\
\hline & & & & & $-0.56^{b}$ & $-3.34,0.76$ & 0.39 \\
\hline \multirow[t]{3}{*}{ BMI $\left(\mathrm{kg} / \mathrm{mt}^{2}\right)(n=4747)$} & & $n=165$ & $n=4493$ & $\mathrm{n}=89$ & & & \\
\hline & $\begin{array}{l}24.75,24.1 \\
( \pm 4.6)\end{array}$ & $\begin{array}{l}23.5,22.8 \\
( \pm 4.3)\end{array}$ & $24.7,24.1( \pm 4.6)$ & $27.2,26.6( \pm 4.8)$ & $0.39^{a}$ & $-0.98,1.75$ & 0.99 \\
\hline & & & & & $-1.47^{b}$ & $-2.14,-0.79$ & $<0.001^{* *}$ \\
\hline \multicolumn{8}{|c|}{ Breastfeeding practices and other infant feeding mode variables } \\
\hline \multicolumn{8}{|l|}{ Currently BF (n, \%) } \\
\hline Yes & $3419(68)$ & $60(81.1)$ & $3152(67.6)$ & $207(70.6)$ & $0.19^{a}$ & $0.14,0.27$ & $0.02^{*}$ \\
\hline No & $1611(32)$ & $14(18.9)$ & $1511^{`}(32.4)$ & $86(29.4)$ & $1.15^{b}$ & $0.89,1.49$ & 0.27 \\
\hline \multicolumn{2}{|l|}{ EBF in 0 to 6 months $(n, \%)(n=1338)$} & $n=34$ & $n=1211$ & $n=93$ & & & \\
\hline Yes & $460(34.4)$ & $15(44.1)$ & $410(33.9)$ & $35(37.6)$ & $1.54^{\mathrm{a}}$ & $0.77,3.1$ & 0.21 \\
\hline No & $878(65.6)$ & $19(66.1)$ & $801(66.1)$ & $58(62.4)$ & $1.18^{b}$ & $0.76,1.82$ & 0.45 \\
\hline \multicolumn{2}{|l|}{ Predominant BF $(n, \%)(n=2492)$} & $n=36$ & $n=2313$ & $n=143$ & & & \\
\hline Yes & $127(5.1)$ & $2(5.6)$ & $118(5.1)$ & $7(4.9)$ & $1.09^{\mathrm{a}}$ & $0.26,3.62$ & 0.71 \\
\hline No & $2365(94.9)$ & $34(94.4)$ & $2195(94.9)$ & $136(95.1)$ & $1.04^{b}$ & $0.49,2.18$ & 0.55 \\
\hline \multirow{3}{*}{$\begin{array}{l}\text { Duration of BF (months) } \\
\text { (mean, SD) }(n=1599)\end{array}$} & & $n=38$ & $n=1526$ & $n=35$ & & & \\
\hline & $7.9,( \pm 5.1)$ & $6.9,7( \pm 4.5)$ & $8,7( \pm 5.1)$ & $5.9,6( \pm 4.4)$ & $3.3^{\mathrm{a}}$ & $-0.20,6.81$ & 0.07 \\
\hline & & & & & $0.29^{b}$ & $-0.34,0.38$ & 0.99 \\
\hline \multirow{3}{*}{$\begin{array}{l}\text { Age of introduction of liquids } \\
\text { including formula (months) } \\
\text { (mean, SD) }(n=4927)\end{array}$} & & $n=176$ & $n=4659$ & $n=92$ & & & \\
\hline & 2.6, $( \pm 2.5)$ & $2.5,2( \pm 2.6)$ & $2.6,2( \pm 2.5)$ & $2.4,2.5( \pm 2.5)$ & $0.75^{a}$ & $0.06,1.45$ & $0.03^{*}$ \\
\hline & & & & & $0.02^{b}$ & $-0.34,0.38$ & 0.99 \\
\hline \multirow{3}{*}{$\begin{array}{l}\text { Age of introduction of semisolids } \\
\text { (months) (mean, SD) }(n=4470)\end{array}$} & & $n=149$ & $n=4236$ & $n=85$ & & & \\
\hline & 4.9, $( \pm 2.1)$ & $5.1,5( \pm 2.6)$ & $4.9,5( \pm 2.1)$ & $4.9,5( \pm 1.7)$ & $0.71^{\mathrm{a}}$ & $0.05,1.37$ & $0.03^{*}$ \\
\hline & & & & & $0.07^{b}$ & $-0.25,0.39$ & 0.99 \\
\hline \multirow{3}{*}{$\begin{array}{l}\text { Age of introduction of solids (months) } \\
\text { (mean, SD) }(n=3919)\end{array}$} & & $n=127$ & $n=3713$ & $n=79$ & & & \\
\hline & $7.5,( \pm 2.5)$ & $7.7,8( \pm 2.9)$ & $7.5,7( \pm 2.5)$ & $7.5,7( \pm 2.4)$ & $0.53^{a}$ & $-0.35,1.4$ & 0.44 \\
\hline & & & & & $0.11^{b}$ & $-0.3,0.53$ & 0.99 \\
\hline \multicolumn{2}{|l|}{ Formula feeding $(n, \%)(n=4464)$} & $n=67$ & $n=4141$ & $n=256$ & & & \\
\hline Yes & $1877(42)$ & 34 (45.9) & 1737 (41.9) & $111(43.4)$ & $0.95^{\mathrm{a}}$ & $0.58,1.53$ & 0.90 \\
\hline No & $2587(58)$ & $40(54.1)$ & 2404 (58.1) & $145(56.6)$ & $1.06^{b}$ & $0.82,1.37$ & 0.69 \\
\hline
\end{tabular}

Comparisons performed with $x^{2}$ test or Kruskall-Wallis test or ANOVA (maternal height) as appropriate

${ }^{\text {a } C o m p a r i s o n s ~ b e t w e e n ~ n o r m a l ~ a n d ~ w a s t i n g ~}$

${ }^{b}$ Comparisons between normal and overweight

* Significance at the level of $p<0.05$

** Significance at the level of $p<0.001$ 


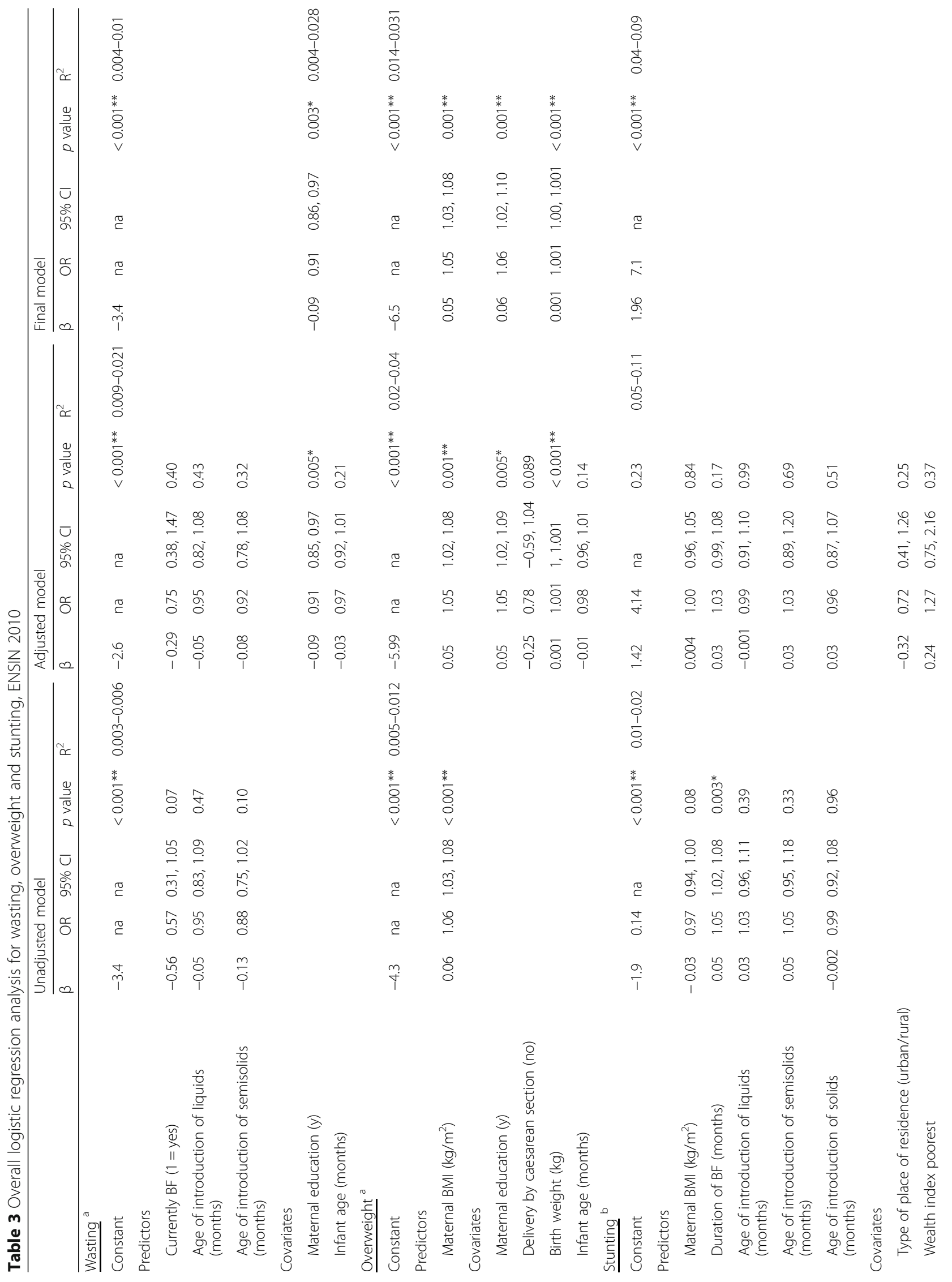




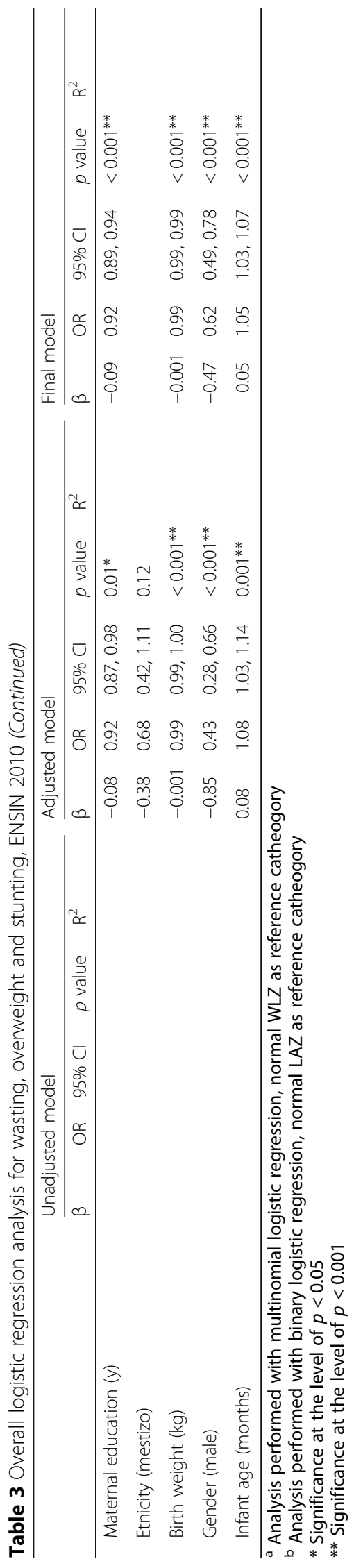


years of maternal education $(\beta=0.06 ; \mathrm{OR}=1.05 ; 95 \%$ $\mathrm{CI}=1.02,1.10)$ and higher birth weight $(\beta=0.001$; $\mathrm{OR}=$ $1.001 ; 95 \% \mathrm{CI}=1.00,1.001$ ) were significant independent predictors of overweight, explaining $4 \%$ of this category $\left(R^{2}=0.04\right)$. However, delivery by caesarean section and infant age were no longer related to overweight (Table 3).

\section{Predictors of stunting}

Compared to non-stunted infants, those with stunting had mothers with lower weight (Mean dif. $=-4.5 \mathrm{~kg}$; $95 \% \mathrm{CI}=-3.5,-5.5)$, shorter height (Mean dif. $=-0.04$ $\mathrm{m} ; 95 \% \mathrm{CI}=-0.03,-0.04)$ and lower BMI (Mean dif. = $\left.0.61 \mathrm{~kg} / \mathrm{m}^{2} ; 95 \% \mathrm{CI}=-0.22,-0.99\right)$; as well as a shorter duration of BF (Mean dif. $=-1.33$; 95\% CI $=-2.06$, 0.60 ) and earlier age of initiation of liquids (Mean dif. $=$ -0.61 months; $95 \% \mathrm{CI}=-0.82,-0.40$ ), semisolids (Mean dif. $=-0.58$ months; $95 \% \mathrm{CI}=-0.77,-0.40)$ and solids (Mean dif. $=-0.61$ months; $95 \% \quad \mathrm{CI}=-0.84, \quad-0.38$ ) (Table 4). Infants who lived in rural areas $(\mathrm{OR}=1.74$; $95 \% \mathrm{CI}=1.47,2.05)$, with lower socioeconomic status $(\mathrm{OR}=1.96 ; 95 \% \mathrm{CI}=1.53,2.49$ for poorer/poorest $)$, with a mother with less years of education (Mean dif. = 2.1 years; $95 \% \mathrm{CI}=-1.78,-2.43)$, belonging to the mestizo ethnic group $(\mathrm{OR}=2.2 ; 95 \% \mathrm{CI}=1.85,2.61)$, with lower birth weight (Mean dif. $=-0.23 \mathrm{~kg} ; 95 \%$ $\mathrm{CI}=-0.17, \quad-0.28)$, male $(\mathrm{OR}=1.27 ; 95 \% \mathrm{CI}=1.08$, 1.51 ) and higher age (Mean dif. $=2.34$ years; $95 \% \mathrm{CI}=$ $0.29,2.77$ ) were more likely to be stunted (Table 5). Maternal height, analysed as a continuous variable, was associated with infant stunting after controlling for potential confounders including as type of place of residence, wealth index, ethnicity and infant gender and age (Mean dif $=0.09 \mathrm{~m} ; 95 \% \mathrm{CI}=0.8,0.11$; data not shown).

The unadjusted binary logistic model showed that none of the anthropometric and other infant feeding mode variables were related to stunting, apart from a longer duration of $\mathrm{BF}(\beta=0.05 ; \mathrm{OR}=1.05 ; 95 \%$ $C I=1.02,1.08)$. When adjusted for covariates, duration of BF was no longer significant. In the final model, stunted infants had mothers with fewer years of education $(\beta=-0.09 ; \mathrm{OR}=0.92 ; 95 \% \mathrm{CI}=0.89$, 0.94), lower birth weight $(\beta=-0.001$; OR $=0.99 ; 95 \%$ $\mathrm{CI}=0.99,0.99)$, were female $(\beta=-0.47 ; \mathrm{OR}=0.43$; $95 \% \mathrm{CI}=0.49,0.78)$ and had higher infant age $(\beta=$ $0.05 ; \mathrm{OR}=1.08 ; 95 \% \mathrm{CI}=1.03,1.07$ ) (Table 3). $\mathrm{EBF}$ in infants up to 6 months did not predict stunting $(\mathrm{OR}=0.75,95 \% \mathrm{CI}=0.14,1.63$; data not shown $)$. The association between infant age and wasting, overweight and stunting was significant in the binary analyses, but only remained significant in the final model for stunting.

\section{Discussion}

To our knowledge, this is the first study in Colombia that describes the associations between maternal BMI or infant feeding mode and the prevalence of infant wasting, overweight and stunting. The main finding was a positive association between maternal BMI and infant overweight after controlling for potential confounders. This finding is in accordance with studies carried out in Colombia in children aged 5 to 12 years that found a relationship between childhood obesity and maternal overweight $(\mathrm{aOR}=2.22 ; 95 \% \mathrm{CI}=1.73$, $2.85)$ and obesity $(\mathrm{aOR}=3.6 ; 95 \% \mathrm{CI}=2.64,4.93)$ [33], and with other Latinamerican studies that show a higher risk of child overweight (>85th percentile) or obesity ( $>95$ th percentile) in those with obese mothers $(\mathrm{OR}=2 ; 95 \% \mathrm{CI}=1.25,3.47$ and $\mathrm{OR}=2.14 ; 95 \% \mathrm{CI}=$ $1.12,4.08)$ [34]. Although it was not possible to evaluate the relationship between maternal weight during pregnancy and gestational weight gain or infant weight change with infant overweight, our results could be interpreted as suggesting an association between maternal and infant weight which could be initiated during pregnancy or even before conception $[35,36]$. This may reflect a combination of environmental factors and shared genetic factors between mother and infant. In Hispanic mothers, a high maternal genetic obesity risk score is associated with increased fetal weight during the first trimester of pregnancy $(\beta=3.1 \mathrm{~g}, 95 \% \mathrm{CI}=1.1-5.1, \quad P=0.003$ for interaction) [37] and the ancestral genetic background is associated with excess weight in infant under $12 \mathrm{mo}$ $(\mathrm{OR}=3.85 ; 95 \% \mathrm{CI}=1.92-7.70)$ [38].

The relationship between maternal anthropometry or infant feeding mode variables with stunting did not persist in multivariable analyses. However, the association of maternal height with infant stunting suggests that genetic factors may contribute, as reported in a study in Mexico where short maternal stature $(<1,45 \mathrm{~m})$ predicted infant stunting $(\mathrm{OR}=3.9 ; 95 \% \mathrm{CI}=3.2,4.8)$. This association persisted only in some areas of Mexico after adjusting by area of residence, suggesting that a shared adverse environment could also be relevant [39]. Stunted infants were older, most likely due to the cumulative impact of adverse health and nutritional exposures in utero and during the first months of life that prevent the infant from attaining its linear growth potential [40].

Unexpectedly, EBF, BF duration, age of initiation of liquids, semisolids and solids and formula feeding were not associated with infant wasting. Cross-sectional studies have shown an inverse association between breastfeeding and the rate of wasting in low-income countries [41]; in our study we found the same trend between a higher prevalence of $\mathrm{BF}$ and lower prevalence of wasting 
Table 4 Breastfeeding practices compared between LAZ categories, ENSIN 2010

\begin{tabular}{|c|c|c|c|c|c|c|}
\hline & \multirow{2}{*}{$\begin{array}{l}\text { Overall } \\
(n=5030)\end{array}$} & \multicolumn{5}{|l|}{ LAZ } \\
\hline & & $\begin{array}{l}\text { Stunting }<-2 \text { SD } \\
(n=645)\end{array}$ & $\begin{array}{l}\text { Normal } \geq-2 \text { SD } \\
(n=4385)\end{array}$ & $\begin{array}{l}\text { OR } \\
\text { /Difference }\end{array}$ & $95 \% \mathrm{Cl}$ & $p$ value \\
\hline \multicolumn{7}{|l|}{ Maternal anthropometry (mean, SD) } \\
\hline \multirow[t]{2}{*}{ Weight $(\mathrm{kg})(\mathrm{n}=4750)$} & & $n=619$ & $n=4131$ & & & \\
\hline & $59.8,( \pm 12.0)$ & $55.8,54.5( \pm 11.36)$ & $60.30,58.10( \pm 11.9)$ & 4.5 & $3.5,5.5$ & $<0.001^{* *}$ \\
\hline \multirow[t]{2}{*}{ Height $(\mathrm{mt})(n=5384)$} & & $n=619$ & $n=4130$ & & & \\
\hline & $1.55,( \pm 0.06)$ & $1.51,1.51( \pm 0.06)$ & $1.55,1.55( \pm 0.06)$ & 0.04 & $0.03,0.04$ & $<0.001^{* *}$ \\
\hline \multirow[t]{2}{*}{ BMI $\left(\mathrm{kg} / \mathrm{mt}^{2}\right)(n=5384)$} & & $n=619$ & $n=4128$ & & & \\
\hline & $24.8,( \pm 4.6)$ & $24.23,23.63( \pm 4.5)$ & $24.8,24.1( \pm 4.6)$ & 0.61 & $0.22,0.99$ & $0.003^{*}$ \\
\hline \multicolumn{7}{|l|}{ Breastfeeding practices and other infant feeding modes } \\
\hline \multicolumn{7}{|l|}{ Currently BF (n, \%) } \\
\hline Yes & $3419(68)$ & $434(67.2)$ & $2985(68.1)$ & 1.04 & $0.87,1.23$ & 0.64 \\
\hline No & $1611(32)$ & $211(32.7)$ & $1400(31.9)$ & & & \\
\hline $\operatorname{EBF}(n, \%)(n=1338)$ & & $n=110$ & $n=1228$ & & & \\
\hline Yes & $460(34.4)$ & $46(41.8)$ & $414(33.7)$ & 0.71 & $0.48,1.05$ & 0.09 \\
\hline No & $878(65.6)$ & $64(58.2)$ & $814(66.3)$ & & & \\
\hline Predominant BF $(n, \%)(n=2492)$ & & $n=296$ & $n=2196$ & & & \\
\hline Yes & $127(5.1)$ & $20(6.8)$ & $107(4.9)$ & 0.74 & $0.48,1.12$ & 1.16 \\
\hline No & $2365(94.9)$ & $276(93.2)$ & $2089(95.1)$ & & & \\
\hline \multirow[t]{2}{*}{ Duration of BF (months) (mean, SD) $(n=1599)$} & & $n=211$ & $n=1388$ & & & \\
\hline & $7.9,7( \pm 5.1)$ & $9.1,9( \pm 5.2)$ & $7.7,7( \pm 5)$ & -1.33 & $-2.06,-0.60$ & $<0.001^{* *}$ \\
\hline \multirow{2}{*}{$\begin{array}{l}\text { Age of introduction of liquids including formula } \\
\text { (months) (mean, SD) }(n=4927)\end{array}$} & & $n=624$ & $n=4303$ & & & \\
\hline & $2.6,( \pm 2.5)$ & $3.1,3( \pm 2.9)$ & $2.5,2( \pm 2.4)$ & -0.61 & $-0.82,-0.40$ & $<0.001^{* *}$ \\
\hline \multirow{2}{*}{$\begin{array}{l}\text { Age of introduction of semisolids (months) } \\
\text { (mean, SD)( } n=4470)\end{array}$} & & $n=574$ & $n=3896$ & & & \\
\hline & $4.9,( \pm 2.1)$ & $5.5,6(2.4)$ & $4.9,5( \pm 2)$ & -0.58 & $-0.77,-0.40$ & $<0.001^{* *}$ \\
\hline \multirow{2}{*}{$\begin{array}{l}\text { Age of introduction of solids (months) } \\
\text { (mean, SD) }(n=3919)\end{array}$} & & $n=523$ & $n=3396$ & & & \\
\hline & $7.5,( \pm 2.5)$ & $8,8( \pm 2.66)$ & $7.4,7( \pm 2.5)$ & -0.61 & $-0.84,-0.38$ & $<0.001^{* *}$ \\
\hline Formula feeding $(n, \%)(n=4464)$ & & $n=550$ & $n=3914$ & & & \\
\hline Yes & $1887(42)$ & $216(39.3)$ & $1661(42.4)$ & 0.87 & $0.73,1.05$ & 0.16 \\
\hline No & $2587(58)$ & $334(60.7)$ & $2253(57.6)$ & & & \\
\hline
\end{tabular}

Comparisons were performed with $x^{2}$ test, $t$ test (maternal height) or U-Mann Whitney test as appropriate

* Significance at the level of $p<0.05$

** Significance at the level of $<0.001$

which could be clinically important although not significant. Maternal recall bias, difficulties in evaluating and defining complex variables such as EBF and duration of $\mathrm{BF}$ could be reasons for the lack of associations with infant anthropometry. Also, the ENSIN survey only recorded information about EBF in infants up to 6 months, so there was a smaller sample for evaluation of the associations EBF and anthropometry. Although the data recorded in ENSIN is evaluated as high quality by DHS based on missing values and inconsistencies; this does not necessarily exclude inaccuracies in the data collected [42], mainly due to the measurement in the home which does not guarantee a flat surface, and the estimation of infant weight (measured with the mother).

A growing body of evidence shows the importance of maternal nutritional status, especially during the perinatal period, on infant nutritional status (The first 1000 days of life) $[21,35,43]$. This association has been reported in studies where prepregnancy BMI had a positive correlation with birth weight, length and head circumference $(\beta=0.274,0.094$ and 0.101 , respectively; $p<0.05$ in all cases) [44]. Also, poor maternal nutritional status predicts undernutrition in 
Table 5 Baseline characteristics compared between LAZ categories, ENSIN 2010

\begin{tabular}{|c|c|c|c|c|c|c|}
\hline & \multirow{2}{*}{$\begin{array}{l}\text { Overall } \\
(n=5030)\end{array}$} & \multicolumn{5}{|l|}{ LAZ } \\
\hline & & $\begin{array}{l}\text { Stunting }<-2 \text { SD } \\
(n=645)\end{array}$ & $\begin{array}{l}\text { Normal } \geq-2 \text { SD } \\
(n=4385)\end{array}$ & $\begin{array}{l}\text { OR } \\
\text { /Difference }\end{array}$ & $95 \% \mathrm{Cl}$ & $p$ value \\
\hline \multicolumn{7}{|c|}{ Sociodemographic characteristics (n, \%) } \\
\hline \multicolumn{7}{|c|}{ Type of place of residence } \\
\hline Rural & $1841(36.6)$ & $311(48.2)$ & $1530(34.9)$ & 1.74 & $1.47,2.05$ & $<0.001^{* *}$ \\
\hline Urban & $3189(63.4)$ & $334(51.8)$ & $2855(65.1)$ & & & \\
\hline \multicolumn{7}{|l|}{ Wealth index } \\
\hline Poorer and poorest & $3167(63)$ & $502(77.8)$ & $2665(60.8)$ & 1.96 & $1.53,2.49$ & $<0.001^{* *}$ \\
\hline Middle & 945 (18.8) & $83(12.9)$ & $862(19.7)$ & 1 & & \\
\hline Richer and richest & $918(18.3)$ & $60(9.3)$ & $858(19.6)$ & 0.73 & $0.51,1.02$ & 0.07 \\
\hline \multicolumn{7}{|l|}{ Maternal characteristics } \\
\hline Age (y) (mean, SD) & $26.6, \pm 6.3$ & $26.9( \pm 6.6)$ & $26.5( \pm 6.3)$ & -0.39 & $-0.92,0.13$ & 0.25 \\
\hline $\begin{array}{l}\text { Maternal education (y) } \\
\text { (mean, SD) }\end{array}$ & $8.4, \pm 3.9$ & $6.5( \pm 3.9)$ & $8.6( \pm 3.9)$ & 2.1 & $1.78,2.43$ & $<0.001^{* *}$ \\
\hline \multicolumn{7}{|l|}{ Ethnicity (n, \%) } \\
\hline Mestizo & $3624(72)$ & $366(56.7)$ & $3258(74.3)$ & 2.2 & $1.85,2.61$ & $<0.001^{* *}$ \\
\hline Minorities & $1406(28)$ & $279(43.3)$ & $1127(25.7)$ & & & \\
\hline \multicolumn{7}{|l|}{ Currently working (n, \%) } \\
\hline Yes & $2047(40.7)$ & $293(45.4)$ & $1754(40)$ & 1.24 & $1.06,1.47$ & $0.009^{*}$ \\
\hline No & $2983(59.3)$ & $352(54.6)$ & $2631(60)$ & & & \\
\hline \multicolumn{7}{|c|}{ Mother lives with the partner (n, \%) } \\
\hline Yes & $3816(75.9)$ & $499(77.4)$ & $3317(75.6)$ & 1.1 & $0.90,1.34$ & 0.34 \\
\hline No & $1214(24.1)$ & $146(22.6)$ & $1068(24.4)$ & & & \\
\hline \multicolumn{7}{|l|}{ Pregnancy conditions } \\
\hline $\begin{array}{l}\text { Alcohol at pregnancy } \\
(n, \%)(n=4942)\end{array}$ & & $n=625$ & $n=4317$ & & & \\
\hline Yes & $451(9)$ & $46(7.4)$ & $405(9.4)$ & 0.76 & $0.56,1.05$ & 0.1 \\
\hline No & $4491(89.3)$ & $579(92.6)$ & 3912 (90.6) & & & \\
\hline $\begin{array}{l}\text { Smoked at pregnancy } \\
(n, \%)(n=4942)\end{array}$ & & $n=625$ & $n=4317$ & & & \\
\hline Yes & $105(2.1)$ & $13(2.1)$ & $92(2.1)$ & 0.97 & $0.54,1.75$ & 0.93 \\
\hline No & 4837 (96.2) & $612(97.9)$ & 4225 (97.9) & & & \\
\hline \multicolumn{7}{|c|}{$\begin{array}{l}\text { Caesarean section delivery } \\
(n, \%)\end{array}$} \\
\hline Yes & 1579 (31.4) & $169(26.2)$ & $1410(32.2)$ & 0.75 & $0.62,0.90$ & $0.002^{*}$ \\
\hline No & $3451(68.6)$ & $476(73.8)$ & 2975 (67.8) & & & \\
\hline \multicolumn{7}{|l|}{ Infant charactetistics } \\
\hline $\begin{array}{l}\text { Birth weight }(\mathrm{kg}) \\
(\text { mean, } S D)(n=3877)\end{array}$ & $3.3, \pm 0.5$ & $3.1( \pm 0.6)$ & $3.3( \pm 0.5)$ & 0.23 & $0.17,0.28$ & $<0.001^{* *}$ \\
\hline \multicolumn{7}{|l|}{ Gender (n, \%) } \\
\hline Male & $2605(51.8)$ & $368(57.1)$ & $2237(51)$ & 1.27 & $1.08,1.51$ & $0.004^{* *}$ \\
\hline Female & $2425(48.2)$ & $277(42.9)$ & $2148(49)$ & & & \\
\hline $\begin{array}{l}\text { Infant age (months) } \\
\text { (mean, SD) }\end{array}$ & $12.1, \pm 7.1$ & $14.2( \pm 6.9)$ & $11.9( \pm 7.0)$ & -2.34 & $-0.29,-2.77$ & $<0.001^{* *}$ \\
\hline
\end{tabular}

Comparisons were performed with $\mathrm{x}^{2}$ test or U-Mann Whitney test (all the continuous variables non-parametric distributed) as appropriate

Reference for all comparisons was normal LAZ

* Significance at the level of $p<0.05$.

** Significance at the level of $p<0.001$. 
infants at 6 and 12 mo [45]. Our study suggests that the association between maternal BMI and infant nutritional status can persist up to $24 \mathrm{mo}$, and maternal BMI is an apparent influential modifiable factor that requires adequate pre and postnatal advice for women.

Maternal education was associated with infant wasting, overweight and stunting, as reported in some other studies where each additional level of education was associated with a $44 \%$ reduction in stunting [46]. In a pooled analysis of five DHS surveys carried out in Bangladesh using data from 1999 to 2011, a lower maternal education level was consistently associated with infant stunting, wasting and underweight across the time-period of study [47]. In low socioeconomic populations, maternal education was inversely associated with child overweight, after controlling for gender [48]. However, we found an increased risk of infant overweight in more highly educated mothers, defined in this cohort as completing elementary and secondary studies but not college or further education. As maternal education could be a proxy for socioeconomic status, this association merits further analysis, including environmental conditions and maternal health-seeking behaviour [49]. Maternal education could protect against infant wasting and stunting if public policy focuses on the empowerment of women and their role in infant development.

The birth weight reflects fetal development and was found to be a significant predictor of infant anthropometry in this secondary analysis. Although one limitation was the fact that birth weight was self-reported by the mother, there was a consistent, positive and strong association between birth weight and overweight and an inverse association with stunting. This finding is in agreement with other studies in which birth weight is associated not only with anthropometry later in life but also with the risk of metabolic syndrome during adolescence $(\mathrm{OR}=1.4 ; 95 \% \mathrm{CI}=1.2-1.6) \quad[50]$ and adulthood, regardless of the presence or absence of gestational diabetes [51].

This study had some limitations, such as the oversampling of mestizo subjects and those with lower and lowest socioeconomic status, which may limit the generalizability of the findings to other Colombian ethnic and socioeconomic groups. Also, the ENSIN 2010 survey did not collect information about important aspects that could influence infant nutritional status such as paternal anthropometric status, preconceptional maternal weight and maternal weight gain during pregnancy. Unfortunately, the EBF variable was constructed using the information on both infant food consumption from the previous $24 \mathrm{~h}$ and data on the age of initiation of liquids other than breast milk, semisolid and solid foods. Inconsistencies in the prevalence of EBF using both methods suggest that mothers may not have understood the concept of $\mathrm{EBF}$ or the questions were inadequate to explore this. BF support at health institutions in Colombia is not well described in the survey, and that lack of information did not allow us to explore associations between BF counselling and duration of BF and EBF. Another limitation was that ENSIN 2010, which provided data for this secondary analysis, is a crosssectional survey which does not permit prospective analysis of the effect of the factors associated with infant anthropometry.

\section{Conclusion}

Greater understanding of factors associated with infant anthropometry during the first 2 years of life is vital in order to influence the risk of both wasting and overweight. In the present secondary analysis, we found a strong association between higher maternal BMI and infant overweight, but no association with EBF, BF and age of initiation of liquids, semisolids and solids. Thus, modifiable factors related to maternal nutritional status such as maternal BMI and maternal weight, are important and plausible targets to develop public policy at the population level that promotes healthy growth in the infant population. Efforts in primary care health to promote healthy maternal weight, starting pre-conception, but continuing during pregnancy, may reduce the risk of overweight and obesity in the offspring and have long-term health benefits.

\section{Abbreviations}

BF: Breastfeeding; BMI: Body Mass Index; EBF: Exclusive Breastfeeding; ENSIN: Encuesta Nacional de la Situación Nutricional; g: Gram; kg: Kilogram; LAZ: Length for Age Z score; mo: Months; w: Week; WAZ: Weight for Age Z score; WHO: World Health Organization; WLZ: Weight for Length Z score; $y$ : Years

\section{Acknowledgements}

Instituto Colombiano de Bienestar Familiar (ICBF).

\section{Authors' contributions}

According to with International Committee of Medical Journal Editors, the following are authors contributions: 1. FAP: conception, design, acquisition of data, analysis and interpretation of data, drafting the work, revising the work critically for important intellectual content. 2. GOV: conception, critical review. 3. MF: critical review. All authors read and approved the final manuscript.

\section{Authors' information}

Fanny Aldana-Parra, Registered Nutritionist \& Dietitian, PhD at the Faculty of Sciences, Pontificia Universidad Javeriana; Clinical Epidemiologist at Universidad El Bosque. Gilma Olaya Vega, PhD, Professor at the Departamento de Nutrición y Bioquímica, Pontificia Universidad Javeriana; MSc in Human Nutrition, PhD in UCL GOS Institute of Child Health, London. Mary Fewtrell, Professor of Paediatric Nutrition \& Hon Consultant Paediatrician. Childhood Nutrition Research Center UCL GOS Institute of Child Health, London. 


\section{Funding}

Not needed.

\section{Availability of data and materials}

The database is available at the Demographic and Health Surveys Program under permission of DHS.

\section{Ethics approval and consent to participate}

During recruitment for the initial study, the researcher explained the study procedures, checked the understanding of participants and answered their questions. Every participant gave written informed consent. For the purpose of the present study, permissions to secondary analysis were obtained from Instituto Colombiano de Bienestar Familiar and from The Demographic and Health Surveys Program.

\section{Consent for publication}

Not applicable.

\section{Competing interests}

The authors declare that they have no competing interests.

\section{Author details}

'Departamento de Nutrición y Bioquímica, Pontificia Universidad Javeriana, Bogotá, Colombia. ${ }^{2}$ GOS Institute of Child Health, University College London, London, UK

Received: 16 October 2019 Accepted: 31 January 2020

Published online: 14 February 2020

\section{References}

1. Barker DJ, Eriksson JG, Forsén T, Osmond C. Fetal origins of adult disease: strength of effects and biological basis. Int J Epidemiol. 2002;31:1235-9.

2. Gluckman PD, Hanson MA, Beedle AS. Early life events and their consequences for later disease: a life history and evolutionary perspective. Am J Hum Biol. 2007;19:1-19.

3. Roseboom TJ, et al. Effects of prenatal exposure to the Dutch famine on adult disease in later life: an overview. Mol Cell Endocrinol. 2001;185:93-8.

4. Lake JK, Power C, Cole TJ. Child to adult body mass index in the 1958 British birth cohort: associations with parental obesity. Arch Dis Child. 1997; 77:376-80.

5. Barnes S, Ozanne S. Pathways linking the early environment to long-term health and lifespan. Prog Biophys Mol Biol. 2011;106:323-36.

6. Bayol SA, Simbi BH, Stickland NC. A maternal cafeteria diet during gestation and lactation promotes adiposity and impairs skeletal muscle development and metabolism in rat offspring at weaning. J Physiol. 2005;567:951-61.

7. Rossiter MD, et al. Breast, formula and combination feeding in relation to childhood obesity in Nova Scotia, Canada. Mater Child Health J. 2015:19: 2048-56.

8. Duijts $L$, Jaddoe W, Hofman A, Moll HA. Prolonged and exclusive breastfeeding reduces the risk of infectious diseases in infancy. Pediatrics. 2010;126:e18-25.

9. Horta BL, Victora CG. Long-term effects of breastfeeding-a systematic review; 2013.

10. Scoccianti $C$, et al. European code against cancer 4th edition: breastfeeding and cancer. Cancer Epidemiol. 2015;39:S101-6.

11. Norat $T$, Chan D, Lau R, Vieira R. The associations between food, nutrition and physical activity and the risk of breast cancer. Washington. DC: World CancerResearch Fund and American Institute for Cancer Research; 2008.

12. Sasamoto, N., et al. Breastfeeding pattern and ovarian cancer risk: Results from the Ovarian Cancer Association Consortium. (AACR), 2019).

13. Martens PJ, et al. Breastfeeding initiation associated with reduced incidence of diabetes in mothers and offspring. Obstet Gynecol. 2016; 128:1095-104.

14. Kramer MS, et al. Effects of prolonged and exclusive breastfeeding on child height, weight, adiposity, and blood pressure at age $6.5 \mathrm{y}$ : evidence from a large randomized trial. Am J Clin Nutr. 2007:86:1717-21.

15. Victora CG, et al. Breastfeeding in the 21st century: epidemiology, mechanisms, and lifelong effect. Lancet. 2016;387:475-90.

16. Instituto Colombiano de Bienestar Familiar: Encuesta Nacional de Situación Nutricional 2015. https://www.minsalud.gov.co/Paginas/Gobierno-presenta-
Encuesta-Nacional-de-Situacion-Nutricional-de-Colombia-ENSIN-2015.aspx. (Accessed 29 Aug 2018.)

17. United Nations Inter-agency Group for Child Mortality Estimation, et al. Levels \& Trends in Child Mortality: Report 2018, Estimates Developed by the United Nations Children's Fund, Geneva, 2018.

18. WHO. Global malnutrition estimates, 2019 edition. Vol. 2019 (http://apps. who.int/gho/tableau-public/tpc-frame.jsp?id=402, 2019).

19. WHO. World Health Statistics 2018: Monitoring Health for the SDGs Sustainable Development Goals. Geneva: World health organization; 2018.

20. Bank, W. Country classification. Vol. 2019 (http://apps.who.int/gho/tableaupublic/tpc-frame.jsp?id=402).

21. Baker JL, Michaelsen KF, Rasmussen KM, Sørensen TI. Maternal prepregnant body mass index, duration of breastfeeding, and timing of complementary food introduction are associated with infant weight gain. Am J Clin Nutr. 2004:80:1579-88.

22. Goldstein RF, et al. Association of gestational weight gain with maternal and infant outcomes: a systematic review and meta-analysis. Jama. 2017: 317:2207-25.

23. ICBF. Encuesta Nacional de la Situación Nutricional en Colombia (ENSIN): Instituto Nacional de Salud, Instituto Colombiano de Bienestar Familiar; Bogotá, Colombia, 2010

24. DANE. Estadísticas por tema: Demografía y población. (2017).

25. Samper B, et al. Encuesta Nacional de la situación nutricional en Colombia ENSIN 2005. In: Instituto Colombiano de Bienestar Familiar. República de Colombia; 2005. p. 65-6.

26. de Onis M, Habicht J-P. Anthropometric reference data for international use: recommendations from a World Health Organization expert committee. Am J Clin Nutr. 1996;64:650-8.

27. WHO. Training course on child growth assessment. Geneva: World Health Organization; 2008.

28. de Onis, M., et al. WHO child growth standards: length/height-for-age, weight-for-age, weight-for-length, weight-for-height and body mass indexfor-age-methods and development. (2006).

29. WHO. Global strategy for infant and young child feeding. Geneva: UNICEF World Health Organization; 2003.

30. Rutstein S. Steps to constructing the new DHS Wealth Index. Rockville: ICF International; 2015.

31. Hood MY, et al. Parental eating attitudes and the development of obesity in children. The Framingham Children's Study. Int J Obesity. 2000;24:1319.

32. Whitaker $\mathrm{KL}$, Jarvis MJ, Beeken RJ, Boniface D, Wardle J. Comparing materna and paternal intergenerational transmission of obesity risk in a large population-based sample. Am J Clin Nutr. 2010;91:1560-7.

33. McDonald CM, Baylin A, Arsenault JE, Mora-Plazas M, Villamor E. Overweight is more prevalent than stunting and is associated with socioeconomic status, maternal obesity, and a snacking dietary pattern in school children from Bogota, Colombia. J Nutr. 2009;139:370-6.

34. Hernández-Valero MA, et al. Maternal BMI and country of birth as indicators of childhood obesity in children of Mexican origin. Obesity. 2007;15:2512-9

35. Sunsaneevithayakul $P$, et al. Relation between gestational weight gain and pregnancy outcomes. J Obstet Gynaecol Res. 2014;40:995-1001.

36. Dennison B, Edmunds LS, Stratton HH, Pruzek RM. Rapid infant weight gain predicts childhood overweight. Obesity. 2006;14(3):491-99.

37. Shrestha D, Rahman ML, Hinkle SN, Workalemahu T, Tekola-Ayele F. Maternal BMl-increasing genetic risk score and fetal weights among diverse US ethnic groups. Obesity. 2019;27:1150-60.

38. Hazrati $\mathrm{S}$, et al. Association of Ancestral Genetic Admixture and Excess Weight at Twelve Months of Age. Child Obes. 2020;16(1): 59-64.

39. Hernandez-Diaz $\mathrm{S}$, et al. Association of maternal short stature with stunting in Mexican children: common genes vs common environment. Eur J Clin Nutr. 1999:53:938

40. Dewey KG, Begum K. Long-term consequences of stunting in early life. Matern Child Nutr. 2011:7:5-18.

41. Talukder A. Risk factors associated with wasting among under-5 children residing in urban areas of Bangladesh: a multilevel modelling approach. J Public Health (Berl.) (2019). https://doi.org/10.1007/s10389019-01163-4.

42. Pullum TW. An assessment of the quality of data on health and nutrition in the DHS surveys 1993-2003: 2008.

43. Barker DJ. The fetal and infant origins of adult disease. BMJ. 1990;301:1111. 
44. Bhargava A. Modeling the effects of maternal nutritional status and socioeconomic variables on the anthropometric and psychological indicators of Kenyan infants from age 0-6 months. Am J Phys Anthropol. 2000;111:89-104.

45. Medhin $\mathrm{G}$, et al. Prevalence and predictors of undernutrition among infants aged six and twelve months in Butajira, Ethiopia: the P-MaMiE birth cohort. BMC Public Health. 2010;10:27.

46. Frost MB, Forste R, Haas DW. Maternal education and child nutritional status in Bolivia: finding the links. Soc Sci Med. 2005;60:395-407.

47. Hasan MT, Soares Magalhaes RJ, Williams GM, Mamun AA. The role of maternal education in the 15-year trajectory of malnutrition in children under 5 years of age in B angladesh. Matern Child Nutr. 2016;12:929-39.

48. Soskolne V, Cohen-Dar M, Obeid S, Cohen N, Rudolf MCJ. Risk and protective factors for child overweight/obesity among low socio-economic populations in Israel: a cross sectional study. Front Endocrinol. 2018;9:456.

49. Desai S, Alva S. Maternal education and child health: is there a strong causal relationship? Demography. 1998;35:71-81.

50. Gillman MW, Rifas-Shiman S, Berkey CS, Field AE, Colditz GA. Maternal gestational diabetes, birth weight, and adolescent obesity. Pediatrics. 2003; 111:e221-6.

51. Ylihärsilä $\mathrm{H}$, et al. Birth size, adult body composition and muscle strength in later life. Int J Obes. 2007:31:1392-9.

\section{Publisher's Note}

Springer Nature remains neutral with regard to jurisdictional claims in published maps and institutional affiliations.

Ready to submit your research? Choose BMC and benefit from:

- fast, convenient online submission

- thorough peer review by experienced researchers in your field

- rapid publication on acceptance

- support for research data, including large and complex data types

- gold Open Access which fosters wider collaboration and increased citations

- maximum visibility for your research: over $100 \mathrm{M}$ website views per year

At BMC, research is always in progress.

Learn more biomedcentral.com/submissions 